\section{Glenohumeral Internal Rotation Deficit in Adolescent Water Polo Players}

\section{Michael Suszter ${ }^{1}$, Nicolas Vardiabasis ${ }^{1}$, Mollee Smith ${ }^{2}$ and John Schlechter ${ }^{3 *}$}

${ }^{1}$ Department of Orthopaedic Surgery, Riverside County Regional Medical Center, CA, USA

${ }^{2}$ Department of Rehabilitation, Children's Hospital Orange County, Orange, CA, USA

${ }^{3}$ Adult and Pediatric Orthopedic Specialists, Children's Hospital Orange County, Orange, CA, USA

\begin{abstract}
Objective: To determine the prevalence of Glenohumeral Internal Rotation Deficit (GIRD) in adolescent water polo players.

Design: Cross-sectional study.

Setting: High-School Pool Deck.

Patients/Participants: 21 high school athletes.

Main outcome measures: Pre-examination questionnaire, DASH survey and physical examination measuring rotation of the dominant and non-dominant shoulder were completed.

Results: Average internal rotation deficit for the dominant shoulder was $16.5 \pm 5.9$ with three participants $(14 \%)$ meeting the definition of GIRD with an internal rotation difference of $25^{\circ}$ or more. 13 participants $(62 \%)$ met the total arc definition of GIRD (greater that 10 arc difference when comparing dominant to non-dominant shoulders). The mean DASH score for our study population was $6.3 \pm 8.6$. Analysis was performed and the variables of age, years playing, hours swimming, preseason training hours, and throws per week were evaluated for any relationship to glenohumeral internal rotation. None of the above variables had any significant relationship to the development of GIRD, but preseason-training hours per week approached significance $(p=0.09)$.

Conclusion: Our data revealed that $14 \%$ of the participants met the definition for GIRD. Preseason training hours approached significance for the development of GIRD, suggesting that higher repetitions of overheard throwing may lead to a decrease in glenohumeral internal rotation. Evaluation of the DASH scores

*Corresponding author: John Schlechter, Adult and Pediatric Orthopedic Specialists, Children's Hospital Orange County, 1310 West Stewart Drive, Suite 508, Orange, CA, USA, Tel: +1 7146332111; Fax: +1 7146335615; E-mail: info@youthsportsortho.com
\end{abstract}

Citation: Suszter M, Vardiabasis N, Smith N, Schlechter J (2015) Glenohumeral Internal Rotation Deficit in Adolescent Water Polo Players. J Orthop Res Physiother 1: 006.

Received: March 13, 2015; Accepted: May 10, 2015; Published: May 25, 2015 revealed no association between the players with GIRD. Proper preparation, identification of rotational limitations, and treatment of players with GIRD is important to help prevent potential injuries.

Keywords: Glenohumeral Internal Rotation Deficit - GIRD; Overhead athlete; Shoulder; Thrower's shoulder; Water polo

\section{Introduction}

The glenohumeral joint consists of dynamic and static stabilizer, which works, in concert to allow for stability and mobility through a large arc of motion, in recent years there has been significant focus on shoulder motion, particularly in overhead throwing athletes. Many articles have evaluated both adolescent and adult athletes in sports such as baseball, tennis, swimming, handball and volleyball for the prevalence of Glenohumeral Internal Rotation Deficiency (GIRD), its etiology and subsequent prevention and/or treatment [1-5], Several theories have been proposed, including boney and soft tissue changes [6-9]. It has been shown that appropriate recognition, training, and therapy can stop the progression of GIRD and decrease the prevalence of injuries [4,10-12]. Failure to recognize GIRD can put the shoulder "at risk" for injury, most notably, the posterosuperior labrum, the undersurface of the posterior supraspinatus tendon, and the anterior inferior capsular structures [9,12-14].

Although many well designed studies have evaluated adolescent baseball players and elite water polo players for GIRD $[1,2,14]$, we are not aware of any studies evaluating adolescent water polo players. This study evaluated high school water polo players for shoulder ROM, prevalence of GIRD and various patient specific variables in an effort to delineate potential causes of GIRD in this population. We hypothesized that adolescent water polo players would have GIRD in their throwing arm and a difference in their arc of motion as compared to the contralateral shoulder. In addition, we hypothesized that increasing age, years playing, hours swimming, and number of throws per week would correlate with a higher prevalence of GIRD and a higher Disabilities of the Arm, Shoulder and Hand (DASH) outcome measure $[1,2,4]$.

\section{Materials and Methods}

\section{Participants}

After obtaining institutional review board approval, correspondence elucidating the study was submitted to local area high schools and parents of water polo players. Twenty-one local high school players were voluntarily enrolled in the study. Ages of the participants ranged from 14-18 years with a mean age of 15.7 years. There were 17 males and 4 females, all right hand dominant. For the purposes of this study we did not exclude any participants, as our inclusion criteria were athletes less than 18 years old, uninjured, present and practicing at the day of our visit to the pool deck.

\section{Testing}

Prior to physical examination each player was provided with a questionnaire to determine: age, years of participation (in water polo), hand dominance, estimated number of throws per week, number of hours swimming per week, and participation in preseason sports 
specific training (which included core exercises and stretching). In addition, each player completed the DASH outcome questionnaire [15]. Although not specific to the overhead athlete, the DASH score is known to be a reliable tool for assessing upper extremity disability and can be applied to the overhead athlete $[3,16]$.

All study participants were examined on the pool deck prior to any stretching or warm-up. Examinations were performed supine and all measurements made with a long arm goniometer by a certified sports medicine physical therapist. The glenohumeral joint was abducted to 90 degrees, the elbow flexed to 90 degrees, and internal and external rotation was measured for both the dominant and non-dominant shoulders. The reference points for measurement were the shaft of the ulna and tip of the olecranon as the center or rotation. Conventional reference points for rotation were followed with 0 degrees indicating neutral position of the forearm (shaft of the ulna perpendicular to the ground). For all measurements, the scapula was stabilized to eliminate scapulothoracic motion and the forearm was held in neutral rotation. Each shoulder was then tested for passive range of motion using standard goniometric technique [17]. A single measurement for both external and internal rotation was done. Maximum rotation was determined by the initiation of scapular motion (Figure 1a and b).

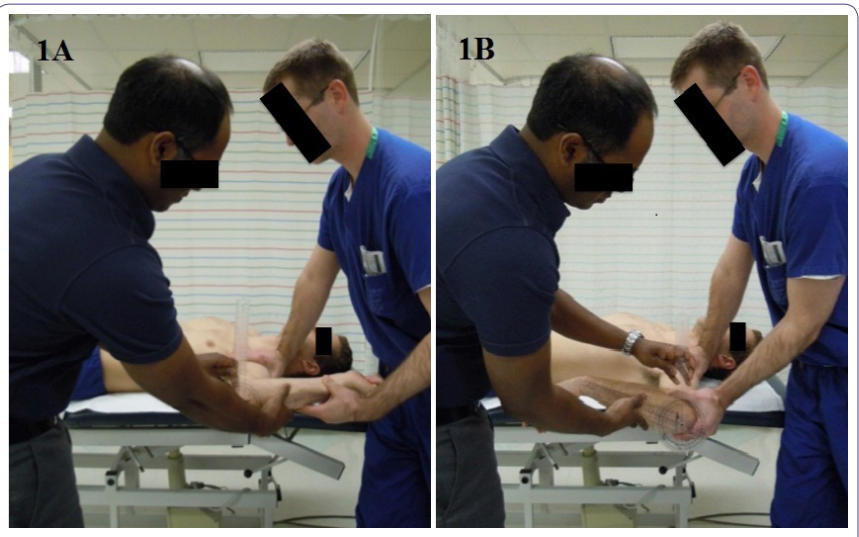

Figure 1a and b: Measurement technique for external and internal rotation.

\section{Definition of GIRD}

Participants were determined to have exhibited GIRD if their throwing shoulders displayed a $25^{\circ}$ or more loss of IR compared with their non throwing shoulders or if there was a greater that $10^{\circ}$ arc difference when comparing dominant to non-dominant shoulders $[13,18]$.

\section{Statistical Analysis}

All statistical comparisons were performed with Statistical Package for the Social Sciences v 12 (SPSS, Inc. Chicago, Illinois). For categorical comparisons, Chi-square or the Fisher's exact test (as appropriate) was utilized to evaluate differences in proportions. For continuous variables, Mann-Whitney $U$ was utilized when the data was not normally distributed or variances were not homogenous. ANOVA was utilized as appropriate. Descriptive statistics (mean, standard deviation, percentages) were calculated. Alpha was set at $\mathrm{p} \leq 0.05$ to declare significance. Total arc range of motion, internal rotation deficit for each shoulder and DASH scores were calculated. Data was analyzed for correlation consisting of a $\mathrm{p} \leq 0.05$ for GIRD and increasing age, years playing, hours swimming, and number of throws per week.

\section{Results}

Twenty-one players in total were evaluated 17 males and 4 females. All participants were right hand dominant. The mean age was $15.7 \pm 0.9$ years (range 14-18). The mean number of years playing was $3.0 \pm 1.4$ years (range 0.5-5). On average the players swim $12.9 \pm 3.1$ hours each week (range 8-20 hours) and spend $1.7 \pm 3$ hours (range 0-10) each week participating in sport specific training. When evaluating throws, $19 \%$ of the participants threw less than 100 balls per week, 57\% threw 101-200 balls per week, 5\% threw 201-300 balls per week, and $19 \%$ of the participants threw greater than 300 balls per week.

Range of motion evaluation of the dominant (throwing) shoulder revealed mean external rotation of $93.6^{\circ} \pm 11.9^{\circ}$ and internal rotation of $40.7^{\circ} \pm 10.9^{\circ}$. Range of motion of the non-dominant shoulder showed mean external rotation of $95.3^{\circ} \pm 10.8^{\circ}$ and internal rotation of $57.3^{\circ} \pm 12.8^{\circ}$. The total arc range of motion for the dominant and non-dominant shoulders was $134.4^{\circ} \pm 14.9^{\circ}$ and $153.1^{\circ} \pm 11.2^{\circ}$ respectively. The mean arc difference when comparing the dominant to non-dominant shoulders was $19.5^{\circ} \pm 9.6^{\circ}$ with the dominant arm having less arc range in all participants. The average internal rotation deficit for the dominant shoulder was $16.5^{\circ} \pm 5.9^{\circ}$ with three participants (14\%) meeting the definition of GIRD $[1-5,9,12,13]$ with an internal rotation difference of $25^{\circ}$ or more. When evaluating the difference in total arc between dominant and non-dominant shoulders, 13 participants (62\%) met the alternative definition of GIRD (greater than $10^{\circ}$ arc difference when comparing dominant to non-dominant shoulders). The mean DASH score for our study population was $6.3 \pm 8.6$.

Analysis was performed and the variables of age, years playing, hours swimming, training hours, and throws per week were analyzed for any relationship to glenohumeral internal rotation. None of the above variables had any correlation with the development of GIRD. However, training hours per week approached significance $(\mathrm{p}=0.09)$ but did not achieve significance based on our alpha value of 0.05 . Furthermore, there was no correlation between athletes with GIRD and an increased DASH score when utilizing the classical GIRD definition.

\section{Discussion}

Numerous past studies [1-5] have evaluated and reported on the rotational differences between the dominant and non-dominant arms in overhead athletes. Many have evaluated athletes that participate in tennis, baseball, swimming, handball and volleyball but we are unaware of any studies evaluating adolescent water polo players. This is the first study to our knowledge evaluating glenohumeral internal rotation deficit in adolescent water polo players. Our examinations showed that $14-62 \%$ of the participants met the definition for GIRD (depending on the definition utilized). We hypothesized that increasing age, years playing, hours swimming, and number of throws per week would correlate with a higher prevalence of GIRD and a higher (DASH) outcome measure. Our study, however, did not find any correlation between any of the studied variables.

Our study focused on adolescent water polo players and the majority of clinical data available for GIRD in the adolescent population frequently focuses on baseball [18-20] (Table 1). The cause of GIRD has yet to be definitively established but theories include either an adaptive or developmental causation. Nakamizo et al., evaluated 25 little league pitchers in a biomechanical study [1]. In 
their study group $40 \%$ of the pitchers measured had GIRD and that these players had increased external rotation in their throwing arm as compared to non-throwing controls. They suggested that the development of GIRD happens earlier than previously thought. Meister et al., also evaluated GIRD in little league players [2]. They examined 294 players and determined that the biggest decline in motion occurred in players between 13-14 years old. In addition, they found that there is a linear decrease in range of motion as compared to age, but did not elucidate the cause.

\begin{tabular}{|c|c|c|}
\hline Study & Number of Patients & Prevalence of GIRD \\
\hline Nakamizo [1] & 25; adolescent/little league pitchers & $40 \%$ \\
\hline Shanley [18] & 33; professional pitchers & $25 \%$ \\
\hline Tokish [19] & 23; professional pitchers & $35-43 \%$ \\
\hline Wilk [20] & 288; professional pitchers & $18 \%$ \\
\hline
\end{tabular}

Table 1: Prevalence of GIRD in overhead athletes.

Stretching, strengthening, and rehabilitation of shoulder dysfunction have been the mainstay for prevention of injury in many athletes. Kibler has written numerous articles outlining the importance for proper evaluation and treatment of shoulder injuries $[10,11]$. Therapy fundamentals include a proximal-to-distal framework with focus on proper scapular function before glenohumeral function. The importance of closed chain axial loading exercises for early shoulder rehabilitation is an essential component of a rehabilitation program. Litner et al., evaluated the effects of a stretching program for professional pitchers with GIRD [4]. The authors felt that GIRD is a soft tissue adaptation and, in their study, found that participation in an internal rotation-stretching program (cross-body posterior capsular stretch) can counteract the development of internal rotation deficit. Izumi et al., performed a cadaveric study on the effectiveness of stretches on the posterior capsule [6]. By evaluating 8 different stretching positions they postulated that internal rotation of the glenohumeral joint at $30^{\circ}$ of elevation in the scapular plane provided the greatest stretch of the middle and lower capsule, while internal rotation at $30^{\circ}$ of extension provided the greatest stretch of the upper and lower capsule.

We recognize that this study has limitations. First, there was no control group. With this additional information, we would be able to determine if our study population had an increased risk for development of GIRD as compared to the non-throwing athlete. Second, our study numbers were small. With a larger population, we might be able to determine if any other parameters would approach or become statistically significant. Thirdly, all of our participants swim for many hours each week. It is difficult to determine if their involvement in swimming has any role in their shoulder examination and further evaluation of age matched swimmers would help determine the role of swimming in the players' development of GIRD. Another limitation of this study is the lack of analysis of intra-rater reliability and maybe a focus of future study. Finally, this was a single day study with a single measurement. This introduces measurement error and could be addressed by doing multiple measurements and averaging the results. Strengths of the study include elimination of measurement error by using a standardized, and well accepted, measurement technique with an evaluator doing the examination and a single sports medicine certified physical therapist measuring the motion. A further strength was the blinding of the evaluators to the players' age, hours swimming, years playing, number of balls thrown per week, hand dominance, hours training, and DASH scores.

\section{Conclusion}

This is the first study to our knowledge evaluating glenohumeral internal rotation deficit in adolescent water polo players. The examinations showed that $14-62 \%$ of the participants met the definition for GIRD (depending on the definition utilized). Analysis of age, years playing, number of balls thrown per week, hours of swimming per week all showed no correlation with the development of GIRD in our population. The cause of GIRD in our population is unknown, however, increasing preseason-training hours approached correlation with GIRD, suggesting a possible causal relationship. In addition, evaluation of the DASH scores revealed no association with the development of GIRD. Further evaluation with a larger group may help elucidate risk factors for the development of GIRD in water polo players.

Previous studies evaluating GIRD in overhead throwing athletes indicate that rotational changes occur early in the athlete's career. In addition, they stress that proper preseason preparation, identification of rotational limitations, and treatment of players with GIRD is important to help prevent injuries. Our study shows that there is a high prevalence of GIRD in adolescent water polo players in similar proportion to other adolescent and elite throwing athletes. The clinical significance of this finding is unclear in our population. It has been shown by others that by initiating internal rotation stretching exercises in overhead throwing athletes that the prevalence of GIRD can be decreased and this may have a role in adolescent water polo players.

\section{References}

1. Nakamizo H, Nakamura Y, Nobuhara K, Yamamoto T (2008) Loss of glenohumeral internal rotation in little league pitcher: a biomechanical study. $J$ Shoulder Elbow Surg 17: 795-801.

2. Meister K, Day T, Horodyski M, Kaminski TW, Wasik MP, et al. (2005) Rotational motion changes in the glenohumeral joint of the adolescent/little league baseball player. Am J Sports Med 33: 693-698.

3. Almeida GP, Silveria PF, Rosseto NP, Barbosa G, Ejnisman B, et al. (2013) Glenohumeral range of motion in handball players with and without throwing-related shoulder pain. J Shoulder Elbow Surg 22: 602-607.

4. Litner D, Mayol M, Uzodinma O, Jones R, Labossiere D (2007) Glenohumeral internal rotation deficits in professional pitchers enrolled in an internal rotation stretching program. Am J Sports Med 35: 617-621.

5. Dines JS, Frank JB, Akerman M, Yocum LA (2009) Glenohumeral internal rotation deficits in baseball players with ulnar collateral ligament insufficiency. Am J Sports Med 37: 566-570.

6. Izumi T, Aoki M, Muraki T, Hidaka E, Miyamoto S (2008) Stretching positions for the posterior capsule of the glenohumeral joint: Strain measurements using cadaver specimens. Am J Sports Med 36: 2014-2022.

7. Branch TP, Lawton RL, lobst CA, Hutton WC (1995) The role of glenohumeral capsular ligaments in internal and external rotation of the humerus. Am J Sports Med 23: 632-637.

8. Bonci CM, Hensal FJ, Torg JS (1986) A preliminary study on the measurement of static and dynamic motion at the glenohumeral joint. Am J Sports Med 14: 12-17

9. Burkhart SS, Morgan CD, Kibler WB (2003) The disabled throwing shoulder: spectrum of pathology part I: pathoanatomy and biomechanics. Arthroscopy 19: 404-420.

10. Kibler WB, McMullen J, Uhl T (2001) Shoulder rehabilitation strategies, guidelines, and practice. Orthopedic Clinics of North Am 32: 527-538.

11. Kibler WB, Livingston B (2001) Closed-chain rehabilitation for upper and lower extremities. J Am Acad Orthop Surg 9: 412-421. 
Citation: Suszter M, Vardiabasis N, Smith N, Schlechter J (2015) Glenohumeral Internal Rotation Deficit in Adolescent Water Polo Players. J Orthop Res Physiother 1: 006.

12. Burkhart SS, Morgan CD, Kibler WB (2003) The disabled throwing shoulder: spectrum of pathology. Part II: evaluation and treatment of SLAP lesions in throwers. Arthroscopy. 19: 531-539.

13. Burkhart SS, Morgan CD, Kibler WB (2003) The disabled throwing shoulder: spectrum of pathology part III: the SICK scapula, scapular dyskinesis, the kinetic chain, and rehabilitation. Arthroscopy 19: 641-661.

14. Giombini A, Rossi F, Pettrone FA, Dragoni S (1997) Posterosuperior glenoid rim impingement as a cause of shoulder pain in top level waterpolo players. $J$ Sports Med Phys Fitness 3: 273-278.

15. Hudak PL, Amadio PC, Bombardier C (1996) Development of an upper extremity outcome measure: the DASH (disabilities of the arm, shoulder and hand) [corrected] Upper Extremity Collaborative Group (UECG). Am J Ind Med 29: 602-608.

16. Alberta FG, ElAttrache N, Bissell S, Mohr K, Browdy J, et al. (2010) The development and validation of a functional assessment tool for the upper extremity in the overhead athlete. Am J Sports Med 38: 903-911.
17. Norkin CC, White DJ (2003) Measurement of Joint Motion: A Guide to Goniometry. FA Davis Co, Philadelphia, PA, USA.

18. Shanley E, Thigpen CA, Clark JC, Wyland DJ, Hawkins RJ, et al. (2012) Changes in passive range of motion and development of Glenohumeral Internal Rotation Deficit (GIRD) in the professional pitching shoulder between spring training in two consecutive years. J Shoulder Elbow Surg 21: 16051612.

19. Tokish JM, Curtin MS, Kim YK, Hawkins RJ, Torry MR (2008) Glenohumeral internal rotation deficit in the asymptomatic professional pitcher and its relationship to humeral retroversion. J Sports Sci Med 7: 78-83.

20. Wilk KE, Macrina LC, Fleisig GS, Aune KT, Porterfield RA, et al. (2014) Deficits in glenohumeral passive range of motion increase risk of elbow injury in professional baseball pitchers. Am J Sports Med 42: 2075-2081. 